\title{
Feedback mechanisms between precipitation and dissolution reactions across randomly heterogeneous conductivity fields
}

\author{
Yaniv Edery $^{1 *}$, Martin Stolar ${ }^{1}$, Giovanni Porta $^{2}$, Alberto Guadagnini $^{2}$ \\ ${ }^{1}$ Faculty of Civil and Environmental Engineering, Technion, Haifa, Israel \\ ${ }^{2}$ Department of Civil and Environmental Engineering, Politecnico di Milano, 20133, Milan, Italy; \\ * Corresponding author: Yaniv Edery (yanivedery@technion.ac.il) \\ Highlights \\ - Regions of prevailing precipitation and dissolution are related to preferential flow patterns \\ - Large changes in non-Fickian transport parameters are observed while velocity variance \\ display modest variations \\ - Initial heterogeneity facilitates attaining asymptotic average solute velocity value
}

\begin{abstract}
Our study investigates interplays between dissolution, precipitation, and transport processes taking place across randomly heterogeneous conductivity domains and the ensuing spatial distribution of preferential pathways. We do so by relying on a collection of computational analyses of reactive transport performed in two-dimensional systems where the (natural) logarithm of conductivity is characterized by various degrees of spatial heterogeneity. Our results document that precipitation and dissolution jointly take place in the system, the latter mainly occurring along preferential flowpaths associated with the conductivity field, the former being observed at locations close to and clearly separated from these. High conductivity values associated with the preferential flowpaths tend to further increase in time, giving rise to a self-sustained feedback between transport and reaction processes. The clear separation between regions where dissolution or precipitation takes place is imprinted onto the sample distributions of conductivity which tend to become visibly left skewed with time (with the appearance of a bimodal behavior at some times). The link between conductivity changes and reaction-driven processes promotes the emergence of non-Fickian effective transport features. The latter can be captured through a continuous time random walk model where solute travel times are approximated with a truncated power law probability distribution. The parameters of such a model shift towards values associated with increasingly high non-Fickian effective transport behavior as time progresses.
\end{abstract}

\section{Introduction}

Diagnosis and characterization of the feedback between geochemical precipitation/dissolution reactions and solute transport processes in heterogeneous subsurface systems is key to a variety of environmental and Earth science scenarios (Rege \& Fogler, 1989; Berkowitz et al., 2016). A critical challenge is the emergence of complex dependencies between physical and chemical processes taking place across aquifer bodies (Saripalli et al., 2001). Heterogeneity of these systems promotes diverse patterns of precipitation and/or dissolution that may imprint a variety of dynamic system responses, including, e.g., wormholing and oscillatory behaviors of system attributes such as porosity and 
permeability (Edery et al., 2011; Garing et al., 2015; Golfier et al., 2002). Examples of practical applications in this context include geologic $\mathrm{CO}_{2}$ storage (e.g., Pawar et al., 2015; Noiriel \& Daval, 2017; Cabeza et al., 2020 and references therein), acid injection in production wells (e.g., Liu et al., 2017 and references therein), and reactive transport of contaminants (e.g., Ceriotti et al., 2018; Dalla Libera et al., 2020 and references therein).

Computational studies can assist the analysis of patterns of chemical transport across heterogeneous subsurface systems in the presence of precipitation/dissolution phenomena. While requiring an explicit description of the spatial heterogeneity of the system properties (Atchley et al., 2014), routine application of numerical simulations in practical settings is hampered by $(i)$ our limited knowledge of the system attributes, resulting in uncertainty affecting the parameterization of the underlying physical and chemical processes and their variability, and (ii) the computational costs required to properly quantify such uncertainties and their propagation onto environmental quantities of interest. In this context, we rely on an effective approach to characterize the evolution of key features of solute transport in the presence of rock-fluid interactions across a porous medium whose spatially heterogeneous conductivity field is interpreted according to a commonly employed stochastic framework.

A critical element we tackle is related to the analysis of the dynamic feedback between reactive transport and spatially heterogenous distributions of porous media attributes such as hydraulic conductivity. Following prior studies, we start by recognizing that, even under geochemical equilibrium conditions, the spatial heterogeneity of system attributes typically imprints an uneven spatial distribution of regions where chemical reactions take place, local fluctuations of conductivity being key to this element (Edery, Porta, et al., 2016). Further to this, our conceptualization of the setting is grounded on the observation that rendering of transport features in geological formations through effective formulations typically requires embedding non-Fickian features. To this end, we rely on a continuous time random walk (CTRW) upscaled model where solute travel times are approximated with a truncated power law (TPL) probability density function (PDF), hereafter termed CTRW-TPL (Berkowitz et al., 2006). This effective modeling framework is particularly relevant because the emergence of non-Fickian transport features in heterogeneous formations has been observed at diverse scales of observation, including pore-, laboratory- and field-scale scenarios (e.g., Edery et al., 2011; Muljadi et al., 2018; Menke et al., 2018 and references therein).

In line with our objective, we rest on the framework of analysis developed in (Edery et al., 2014; Edery, Porta, et al., 2016), where an effective depiction of transport processes is parametrized as a function of the statistics of solute residence times in randomly heterogeneous conductivity fields. A main element of this framework is that it yields a link between the CTRW-TPL formulation and the occurrence of preferential pathways that can be obtained from computational studies of transport in such conductivity fields (Edery et al., 2014). As a result, the methodology is conducive to an effective (or upscaled) representation of local features to identify signatures of non-Fickian transport and embed these in the representation of solute breakthrough curves (BTC) as a function of the underlying system properties (see also, e.g., Dentz et al., 2011; Edery et al., 2014). To illustrate the main features associated with the scenario of interest, we consider a Darcy-scale formulation of a reactive transport setup, where precipitation and/or dissolution of minerals are driven by the injection of an acid compound establishing local equilibrium with the resident fluid and a solid matrix of the host porous medium which is considered to be composed of calcite mineral. While the geochemical processes we consider are somehow streamlined with respect to a field-scale scenario, they embed the main elements characterizing the interplay between solute transport and rock-fluid interactions in Darcy-scale systems. Within this conceptual picture, our study aims at investigating $(i)$ the interplay 
between the reactive process and the ensuing spatial distribution of preferential pathways associated with spatially heterogeneous conductivities and ( $i i)$ the link between locally occurring reaction-driven phenomena and emerging non-Fickian effective transport features, as captured by the CTRW-TPL framework.

\section{Methodology}

\subsection{Chemical model}

We simulate a reactive transport scenario where calcite $\left(\mathrm{CaCO}_{3}(s)\right.$, subscript $(s)$ denoting solid mineral) can dissolve or precipitate locally in the presence of chemical equilibrium between dissolved carbonic acid $\left(\mathrm{H}_{2} \mathrm{CO}_{3}\right)$ and $\mathrm{pH}$. The amount of dissolved $\mathrm{H}_{2} \mathrm{CO}_{3}$ as a function of $\mathrm{pH}$ (see Figure $\mathrm{S} 1$ in the supplementary material) is then governed by equilibrium conditions, which is tantamount to assuming a locally instantaneous reaction (i.e., infinite local Damköhler number). The formulation describing the chemical reactions can then be streamlined as:

$\mathrm{CaCO}_{3(s)} \leftrightarrow \mathrm{Ca}^{2+}+\mathrm{CO}_{3}^{2-}$

$\mathrm{H}_{2} \mathrm{CO}_{3} \leftrightarrow 2 \mathrm{H}^{+}+\mathrm{CO}_{3}^{2-}$

according to which two protons $\mathrm{H}^{+}$in (1b) react with $\mathrm{CO}_{3}^{2-}$ to produce $\mathrm{H}_{2} \mathrm{CO}_{3}$ that in turn drives dissolution of the host calcium carbonate solid matrix. In this context, and consistent with typical experimental practice, we consider the injected fluid and the porous medium to be associated with a source of $\mathrm{H}^{+}$and an abundance of $\mathrm{Ca}^{2+}$, respectively. Thus, $\mathrm{Ca}^{2+}$ is not rate limiting and the spatial distribution of $\mathrm{H}^{+}$, as driven by transport and reaction, governs $\mathrm{pH}$. The rate limiting reaction is then (1b), that is controlled by the available $H^{+}$(or $\left.\mathrm{pH}\right)$, similar to observations associated with other studies (Singurindy \& Berkowitz, 2004; Edery et al., 2011). The chemical reaction system (1a) and (1b) is here simplified (see, e.g., Krauskopf \& Bird, 1967) through:

$c o \leftrightarrow 2 h+c$

where $\mathrm{co}$ denotes $\mathrm{H}_{2} \mathrm{CO}_{3}, h$ and c representing $\mathrm{H}^{+}$and $\mathrm{CaCO}_{3}(\mathrm{~s})$, respectively.

\subsection{Flow and transport modeling}

Our computational setting is intended to mimic a laboratory scale scenario where a $60 \times 24 \mathrm{~cm}^{2}$ two-dimensional flow cell is filled with a porous system formed by a $\mathrm{CaCO}_{3}(s)$ solid matrix. The system is initially fully saturated with water and an injection of low $\mathrm{pH}$ water takes place across the upstream side of the cell. To investigate the influence of the dissolution/precipitation reaction on solute transport, we consider a uniform in the mean groundwater flow, taking place within a twodimensional domain where the (natural) logarithm of conductivity, $y=\ln (k)$, is considered as a zero-mean, second-order stationary random field. The latter is further characterized by an isotropic, simple exponential, covariance function, with (normalized) correlation length $l / L, L$ being the length of the domain along the main flow direction. Various degrees of heterogeneity of the system are analyzed upon considering values of log-conductivity variance $\sigma_{0}^{2}=[1,3,5]$, subscript 0 denoting that these values refer to the initially generated conductivity distributions (i.e., prior to the occurrence of reactions). The domain is discretized through $300 \times 120$ elements of uniform size $\Delta=0.2 \mathrm{~cm}$, yielding a field size of $60 \times 24 \mathrm{~cm}^{2}$. Each field is synthetically generated through the widely tested sequential Gaussian simulator GCOSIM3D (Gómez-Hernández \& Journel, 1993) and is characterized by $l / L=0.016$. This yields a value of $\Delta / l=0.2$, which is deemed adequate to capture the local features 
of the covariance of $y$ and their impact on the main statistics of the velocity field and travel times (Ababou et al., 1989; Riva et al., 2009).

For each value of $\sigma_{0}^{2}, 20$ random realizations of $y$ are generated, each being then subject to a deterministic pressure drop $(\Delta H=100 \mathrm{~cm})$ between the inlet (left) and the outlet (right) sides. The local distribution of fluid velocity is computed through

$\nabla \cdot \boldsymbol{q}(\boldsymbol{x})=0 ; \boldsymbol{q}(\boldsymbol{x})=-k(\boldsymbol{x}) \cdot \nabla h(\boldsymbol{x})$

where $\boldsymbol{q}(\boldsymbol{x})$ is the local Darcy flux, vector $\boldsymbol{x}$ corresponding to spatial location. The local fluid velocity field is then obtained as $\boldsymbol{v}=\boldsymbol{q} / \theta$, a constant initial porosity $\theta=0.4$ being here considered for the porous medium.

Solute transport is then simulated across each conductivity field by a particle tracking approach (Le Borgne et al., 2008). A number of $10^{5} \mathrm{~h}$ particles (see (2)), which is selected to represent a full pore volume at constant $\mathrm{pH}=3.5$, is divided by the domain length and multiplied by the mean velocity ( $\hat{\mathbf{v}}$, as evaluated from (4) across the whole domain). A total amount of particles evaluated as Integer $\left(10^{5} / L \cdot \Delta t \cdot \hat{\mathbf{v}}\right)$ is then injected into the system at regular time intervals $(\Delta t=0.1 \mathrm{~min})$. Particles injected at each $\Delta t$ are flux-weighted according to the conductivity distribution at the inlet. The particles representing a full pore volume correspond to 10.79 moles of $H^{+}$, the same amount being injected across the simulation course to obtain a constant $\mathrm{pH}=3.5$ in the injected fluid, while absence of $h$ particles is taken to correspond to $\mathrm{pH}=8$. We then evaluate the $\mathrm{pH}$ value (or $\mathrm{H}^{+}$molar mass) associated with each $h$ particle by dividing the total number of $H^{+}$moles required to obtain a $\mathrm{pH}=3.5$ (i.e., $10.79 \mathrm{~mol}$ of $H^{+}$) by the pore volume (as represented by $10^{5} h$ particles).

The upper and lower boundaries of the domain are reflective while the outlet boundary is absorbing. Transport is simulated through

$\boldsymbol{d}=\boldsymbol{v}\left[\boldsymbol{x}\left(t_{k}\right)\right] \delta t+\boldsymbol{d}_{D}$

where $\boldsymbol{d}$ is particle displacement, $\boldsymbol{x}\left(t_{k}\right)$ is the vector identifying spatial coordinates of particle location at time $t_{k}, v$ is fluid velocity at $\boldsymbol{x}\left(t_{k}\right), \delta t=\delta s / v$ is the temporal displacement magnitude ( $v$ is the norm of $\boldsymbol{v}$ ), and $\boldsymbol{d}_{D}$ is the diffusive displacement. The latter is evaluated as $\boldsymbol{d}_{D}=3 N[0,1] \sqrt{2 D_{m} \delta \mathrm{t}}$, where $N[0,1]$ represents a two-dimensional vector of random variables, whose entries are mutually independent and sampled from a Gaussian distribution with zero mean and unit variance, $D_{m}=10^{-5}$ $\mathrm{cm}^{2} /$ min representing diffusion. The value of $\delta s$ is selected to be an order of magnitude less than $\Delta$, to accurately sample the velocity variability within a conductivity block.

Coupling between particle evolution and the geochemical setup illustrated in Section 2.1 is achieved in two steps. First, we advance all particles according to the displacement mechanism described above. Second, we satisfy the equilibrium condition (2) by equilibrating both co and $h$ within each cell, leading to precipitation or dissolution of a calcite mineral. The calcite volume to mole ratio is taken as $M=37 \frac{\mathrm{cm}^{3}}{\mathrm{~mol}}$ (Morse \& Mackenzie, 1993) and the equilibrium between $h$ and co particles (according to (2)) leads to a local precipitation (or dissolution) of the solid. We update in time the spatial distribution of porosity assuming that it is characterized by a uniform change within each individual domain cell. We finally update conductivity through the Kozeny-Carman (KC) formulation

$k(a r)_{i j}=k(b r)_{i j} \cdot \frac{\theta(a r)_{i j}^{3}}{\left(1-\theta(a r)_{i j}\right)^{2}} \cdot \frac{\left(1-\theta(b r)_{i j}\right)^{2}}{\theta(b r)_{i j}^{3}}$ 
where $k(a r)_{i j}$ and $\theta(a r)_{i j}$ are conductivity and porosity, respectively, after the reaction (ar) has taken place, while $k(b r)_{i j}$ and $\theta(b r)_{i j}$ are their counterparts before the reaction is observed, subscripts $i$ and $j$ being identifiers of a given cell. The process is repeated for each particle in each of the cells until an equilibrium between $c o$ and $h$ is reached. We set an upper and a lower bound of 0.1 and 0.9 , respectively, for porosity, to avoid the occurrence of unphysical porosity values. Precipitation is treated numerically in a corresponding way. We numerically calculate the updated local head and fluid velocity distributions from (4) at time intervals of $10 \Delta t$, to reduce constraints associated with computational costs. The computational cost of each realization is between 1 3 days (depending on the value of $\sigma_{0}^{2}$ ), upon relying on a 16 Xeon $2.6 \mathrm{Ghz}$ processor with 64 GB RAM.

The updated conductivity field is extracted and stored at the above mentioned regular intervals of $10 \Delta t$. Transport of a non-reactive solute pulse is then simulated across each of these updated fields to capture the temporal evolution of the key parameters driving effective transport (see Section 2.3). While noting that natural porous media can exhibit complex relationships between permeability and porosity (Luquot \& Gouze, 2009), which may not always be interpreted through the KC model (6), we employ the latter formulation because it is considered as a reference model in the literature and can serve as a proxy for alternative improved parameterizations (Erol et al., 2017).

\subsection{Quantities of interest}

The workflow described in Section 2.2 enables one to extract computationally-based quantities employed to characterize the analyzed reactive transport setup. As stated in Section 2.2, we simulate a tracer test within the original fields as well as within those modified by precipitation/dissolution. Particles are displaced through the action of advection and diffusion following a pulse (flux-weighted) injection at the inlet. These non-reactive transport simulations are performed to assess base values of parameters characterizing solute transport $(a)$ prior to starting the reactive transport simulation as well as $(b)$ at specific times after reaction changed the field. The empirical PDF of particle waiting times is assessed from the corresponding histogram starting by evaluating particle waiting times within a given domain cell through the inverse of the particle velocity computed at each time step multiplied by the cell length and weighted by the number of particles visiting the cell. This PDF is then used to estimate the parameters of the TPL model

$\psi\left(t_{w}\right)=\frac{n}{t_{1}} \frac{\exp \left(-t / t_{2}\right)}{\left(1+\frac{t}{t_{1}}\right)^{1+\beta}}$

where $t_{w}$ is the waiting time of a particle within a given domain cell, $t_{1}, t_{2}$, and $\beta$ are model calibration parameters, which are estimated through a standard least square technique. Note that previous results have shown that the parameters obtained from (6) can be readily used to interpret breakthrough curves associated with non-reactive solutes (Edery et al., 2014).

The velocity fields are examined upon computing the evolution of the velocity and conductivity fields statistics, as described in the following. Let us consider a discrete field of a generic quantity $z_{i j}$ evaluated in a given cell $i j$. In the particle tracking numerical simulations we quantify $n_{i j}(t)$ as the number of particles that have visited cell $i j$ along the simulation up to a given time $t$. Thus, we evaluate two relative frequency (or empirical probability) distributions, i.e., $f\left(z_{i j}\right)$ and $f\left(n z_{i j}\right)$, hereafter termed as unweighted and weighted distribution of the variable $z_{i j}$, respectively. We define the weighted variable $n z_{i j}(t)=n_{i j}(t) z_{i j} / \bar{n}(t)$, where $\bar{n}$ is the average value of $n_{i j}$. Note that the adopted weighting scheme corresponds to weighting $z_{i j}$ by the solute mass distribution. Average values of the weighted and unweighted distributions (hereafter denoted as $\bar{z}$ and $\bar{n} \bar{z}$, respectively) can 
then be evaluated. In the following we perform particle weighting in the non-reactive as well as in the reactive transport scenarios. Distribution weighting by reactive particles is indicated by $n_{R}$, meaning that weighting is performed based on the reactive transport simulations (i.e., considering $h$ and co particles as explained above). The plain symbol $n$ indicates weighting by non-reactive particles, employed to simulate conservative tracer tests as detailed above. The variable $z_{i j}$ is taken to correspond to either the cell $\log$-conductivity $y_{i j}$ or fluid velocity $v_{i j}$ in the results illustrated in Section 3 .

We start our analyses by simulating transport of a non-reactive tracer across the generated heterogeneous conductivity domains. As log-conductivity variance increases, the range of conductivity values naturally increases, this being reflected in the distribution $f\left(y_{i j}\right)$ (see, e.g., Figure 1 a-c (blue circles)). The shape of weighted conductivity distributions, $f\left(n y_{i j}\right)$, differs from the one of $f\left(y_{i j}\right)$, consistent with the observation that particles are chiefly channelled towards preferential flow pathways. The latter distributions tend to be shifted towards high conductivity values and are characterized by an enhanced mean conductivity value as compared against their generated (unweighted) counterparts (see conductivity mean and weighted mean values in Table 1, and the results corresponding to the blue and red circles depicted in Figure $1 \mathrm{a}-\mathrm{c}$ ). This shift is imprinted onto the probability density function (PDF) of the waiting times and onto its associated CTRW-TPL parameters (see Figure 2 a-c), consistent with prior studies (Edery et al., 2014; Edery, Geiger, et al., 2016; Edery, 2020). We then simulate reactive transport across the collection of generated fields, allowing for precipitation (and/or dissolution) of calcite and assessing the evolution of the conductivity field according to the Kozeny-Carman formulation introduced in Section 2. Conductivity, head, and velocity fields, as well as particle visitations, $n_{i j}(t)$, associated with species $h$ and $c o$ are sampled across time.

After $200 \Delta t$ have elapsed (corresponding to a total simulation time of $20 \mathrm{~min}$, i.e., a full pore volume) a set of $h$ particles connecting the inlet to the outlet of the system is clearly visible (see Figure $3 \mathrm{a}$ and $\mathrm{b}$ ), these particles being non-uniformly distributed in space. Figure $3 \mathrm{a}$ and $\mathrm{b}$ depict a heat map of the $h$ particles distribution at time $t=20 \mathrm{~min}$, clearly evidencing the emergence of regions of preferential flow (PF). We also note that the number of $h$ particles density (corresponding to concentration) tends to decrease with increasing distance from the inlet, these being replaced by $c o$ particles, consistent with the observation that they are consumed during the course of the reactive process which induces dissolution of the host solid matrix. The $h$ and $c o$ particles attain equilibrium within cells away from the inlet. As such, reaction can only take place if a particle leaves (or enters) a cell under the action of advection and/or diffusion leading to a new equilibrium state. When examining the alteration of conductivities due to the dissolution/precipitation reaction, we note that dissolution (corresponding to an increase of permeability values) is primarily tied to the preferential flow pathways. Otherwise, precipitation is seen to take place in regions close (on average) to these pathways. The highest strength of precipitation is observed in the proximity of the preferential pathways, to then decrease with distance from these.

Figures $3 \mathrm{c}$ and e depict the regions where conductivity has increased (due to dissolution) or decreased (due to precipitation), respectively. The $h$ particles invading the domain closely follow the PFs displaying a fingering pattern, leading to a corresponding dissolution pattern associated with locally increased conductivities. Since conductivity values along the PFs are typically higher (on average), dissolution is increasing these conductivities even further, giving rise to a self-sustained 
enhancing mechanism. The concentration of $h$ particles reaches a local (i.e., within a given cell) equilibrium with the produced $c o$ particles. Hence, dissolution will take place where transport induces shifts in concentration that need to be compensated by the dissolution/precipitation process to maintain local equilibrium. Such scenarios can be attained $(i)$ by $c o$ particles exiting the preferential flow pathways due to the action of diffusion (i.e., they leave locations where concentration of $h$ particles is large upon diffusing towards higher $\mathrm{pH}$ regions where they precipitate) or (ii) by $h$ particles traveling through the fast preferential paths and advancing through these. Figure $3 \mathrm{~g}$ and $\mathrm{h}$ display regions with dominating dissolution or precipitation for cells outside and within the PFs, respectively. Here cells associated with PFs are identified upon relying on particle visitations following Edery et al. (2014). Dissolution dominates within the PFs (as indicated by the red cells in Figure $3 \mathrm{~h}$ ), because $h$ particles are injected through a flux-weighted boundary condition. On the other hand, the produced co particles do not precipitate at locations corresponding to the high $h$ concentration residing in the PFs. These may precipitate away from these regions, where they experience low concentrations of $h$ particles. Thus, we observe a reduction of conductivity taking place in regions adjacent to the PFs (Figure $3 \mathrm{~b}$ and $\mathrm{g}$ ). In summary, our computational results document an increase of conductivity along the preferential pathways jointly with a conductivity reduction within regions close to these and along directions approximately normal to them.

Changes of conductivity values ensuing precipitation/dissolution are clearly visible by the broadening of the unweighted $\log$-conductivity distribution $f\left(y_{i j}\right)$, see Figure $1 \mathrm{~d}-\mathrm{f}$, and Figure $1 \mathrm{~g}-\mathrm{i}$ (blue circles), evaluated at times $t=10$ and $t=20 \mathrm{~min}$, respectively. The reaction dynamics leads to a conductivity field characterized by a slightly increased average value, given that our computational analyses entail the injection of an acid fluid into the system (see Table 1 for details). Detailed inspection of Figure 1d and $\mathrm{f}$ reveals that precipitation takes place across a slightly larger area than dissolution, i.e. values of the frequency distribution $f\left(y_{i j}\right)$ associated with low conductivities tend to increase at a larger rate rather than those corresponding to high conductivities (the left tail of the distributions becomes heavier than the right tail with the progress of reaction). The weighted $\left(f\left(n y_{i j}\right)\right)$ and unweighted $\left(f\left(y_{i j}\right)\right)$ distributions (red and blue circles in Figure 1d-f, and Figure $1 \mathrm{~g}$-i at time $t=10$ and $t=20 \mathrm{~min}$, respectively) are visibly broadening, being associated with an average conductivity which is higher than the one of the originally generated conductivity domains (see Table 1). As stated above, dissolution is focused along the preferential pathways, which comprise an area of limited extent with respect to the whole field.

The above documented mechanism and its signature on the weighted and unweighted conductivity frequency distributions are sensitive to the initial log-conductivity variance, $\sigma_{0}^{2}$. When considering both distributions $f\left(y_{i j}\right)$ and $f\left(n y_{i j}\right)$, associated with the case $\sigma_{0}^{2}=1$, the distributions variance are seen to increase in time, as compared to the values attained at the beginning of the simulation (i.e., prior to reaction; see Figure 1a to $\mathrm{d}$ and $\mathrm{g}$, and Table 1). Otherwise, as the initial heterogeneity increases (see, e.g., $\sigma_{0}^{2}=3,5$ ) mean and variance associated with the weighted and unweighted conductivity distributions display only minor changes (approximately 10\%) across the temporal window considered. The conductivity fields characterized by the lowest $\sigma_{0}^{2}$ value are associated with preferential pathways that are not starkly recognizable when analyzed under nonreactive transport conditions. These channels become more clearly distinguishable as reactions induce an increase of the conductivities along the PFs. At the same time, precipitation causes a decrease of the conductivity outside the PF. This leads to an increased importance of the left tail of $f\left(y_{i j}\right)$, corresponding to an increase of low conductivity values (see Figure 1, left middle and bottom rows). 
With reference to the highest conductivity variance analyzed, the reaction patterns for the precipitation and dissolution lead to a smaller relative change between conductivity frequency distributions evaluated prior and after the reaction. Relative changes between the unweighted and weighted conductivity frequency distributions (including the ensuing mean and variance listed in Table 1) evaluated before and after the reaction are less pronounced as the variance of the generated conductivity field increases (see Figure 1 middle and right columns and Table 1). This is related to the observation that, as log-conductivity variance increases, preferential pathways in the originally generated field become markedly more distinct. Thus, relative differences between unweighted and weighted conductivity histograms are seen to diminish in time because the flow field is already organized according to well-identified pathways and tends to preserve its initial pattern (Figure 1d-f, and Figure 1g-i at time $t=10$ and $t=20 \mathrm{~min}$, respectively). Note that low order statistics (i.e., mean and variance) of velocity and conductivity display only a minute evolution with the progress of reaction, in spite of the relevant changes exhibited by the tails of the frequency distributions (see Figure 1) for all considered values of $\sigma_{0}^{2}$, the latter feature being relevant when addressing nonFickian transport, as further discussed below.

As stated in Section 2.2, the conductivity fields altered through precipitation/dissolution and extracted at regular time intervals of $10 \Delta t$ are subject to non-reactive transport analyses and the ensuing evolution of the parameters of the TPL model (6) is analyzed. Key results of these analyses are listed in Table 1 with reference to the original (unaltered) conductivity fields and at the final simulation time (i.e., at time $t=200 \Delta t$ ). Analysis of the results associated with transport across the $\log$-conductivity field characterized by the smallest original variance (i.e., $\sigma_{0}^{2}=1$ ) and listed in Table 1 indicates that the changes of the sample log-conductivity PDF induced by the progress of the reaction are reflected by the parameters of the TPL model (6). These transition from estimated values corresponding to an effective Fickian transport regime (corresponding to $\beta=2$, see also Figure 2a) to values denoting a highly non-Fickian effective transport setting, manifested by the widening of the support of the waiting time PDF $\psi\left(t_{w}\right)$ (see also Figure $2 \mathrm{~d}$ and $2 \mathrm{~g}$, for results obtained at $t=10$ and $20 \mathrm{~min}$, respectively). Effective transport in the domain with the highest variance (i.e., $\sigma_{0}^{2}=5$; see Table 1) is characterized by estimated TPL parameters corresponding to a non-Fickian signature also prior to the occurrence of precipitation/dissolution (Figure 2c). Such a signature is then further enhanced after reaction has altered the conductivity field, yet displaying a less marked evolution of the shape of the $\psi\left(t_{w}\right)$ as compared to the case $\sigma_{0}^{2}=1$ (see also Figure $2 \mathrm{f}$ and i for $t=10$ and $20 \mathrm{~min}$, respectively).

The observed temporal changes in conductivity and the ensuing local dynamics of transport pattern yield global variations in the reaction rate. Consistent with prior studies and the imposed boundary conditions, the mean velocity associated with the originally generated conductivity domains increases with $\sigma_{0}^{2}$. As the reaction progresses and the conductivity fields change, the increased area subject to dissolution leads to a slight increase of the mean velocity for all of the $\sigma_{0}^{2}$ analyzed. To analyze the influence of the preferential flow on the velocity that is affecting particle transport, we consider the average value $\overline{n_{R} v}$, evaluated upon considering weighting by the number of reactive particles, $n_{R}$, visiting each cell (where the term reactive particles denotes both $h$ and $c o$ particles employed in the context of the reactive transport simulations). The weighted average velocity displays an initial increase over time due to the increase of conductivity within the preferential pathways. When considering the relative change across the whole simulation time, values of the temporal increase of $\overline{n_{R} v}$ are similar across the three heterogeneity levels examined, i.e., they are seemingly independent of $\sigma_{0}^{2}$. However, results in Figure $4 \mathrm{a}$ also reveal that the average velocity $\overline{n_{R} v}$ displays distinct temporal histories depending on $\sigma_{0}^{2}$. In particular, the value of $\overline{n_{R} v}$ tends to attain an 
asymptotic value at time $t \approx 7 \mathrm{~min}$ for $\sigma_{0}^{2}=5$, while showing a sustained increasing trend for $\sigma_{0}^{2}=$ 1. This result suggests that the feedback between reaction and flow patterns reaches an asymptotic condition faster in systems characterized by higher heterogeneity.

The temporal evolution of the velocity fields due to the precipitation/dissolution reaction and the resulting conductivity changes lead to time-dependent reaction pattern and reaction rates. The Damköhler number is infinite on a local scale in our computational analyses, because the reaction is instantaneous. When considering the entire system, transport processes induce a net overall reaction rate that can be quantified as the sum of the total conductivity changes across time $\left.\operatorname{Sum}\left(\left|\Delta k_{i j}\right|\right)\left[\frac{\mathrm{cm}}{s e c}\right]\right)$. The latter incorporates both positive and negative changes of hydraulic conductivity and therefore quantifies the overall intensity of precipitation and dissolution processes in the domain. The quantity $\operatorname{Sum}\left(\left|\Delta k_{i j}\right|\right)$ is evaluated across all realizations for each of the $\sigma_{0}^{2}$ values considered and is depicted in Figure $4 \mathrm{~b}$ as a function of time. These results indicate that the overall reaction rate increases in time with a similar rate for all considered values of $\sigma_{0}^{2}$ (figure $4 \mathrm{~b}$ ) at early times. The observed increase is consistent with the initially advancing of the reaction front across the domain. We observe that the reactive processes magnitude is proportional to $\sigma_{0}^{2}$. For low initial levels of heterogeneity, conductivity values along the preferential pathways are closer to the average field conductivity than what can be observed for the highly heterogeneous domains. As such, the portion of the domain where precipitation or dissolution can take place increases at a rate proportional to $\sigma_{0}^{2}$. As the reaction front reaches the domain outlet, the dissolving front found in the PF leaves the domain. Hence, the global variation in conductivity (which is proportional to the magnitude of reactive processes) tends towards an asymptotic value, corresponding to the diffusion-controlled solute exchange along a direction transverse to the preferential pathways (see Figure 3). In agreement with results shown in Figure 4a, this transition towards an asymptotic regime takes place earlier for larger values of $\sigma_{0}^{2}$, while a smaller initial heterogeneity implies a longer transient period.

\section{Conclusions}

Our computational study tackles the quantitative characterization of the feedbacks between precipitation and dissolution reaction dynamics taking place in randomly heterogenous conductivity fields associated with various degrees of spatial heterogeneity. Our results are based on numerical simulations and may be used to inform upscaling approaches to capture the pre-asymptotic and asymptotic dynamics of reactive transport in heterogeneous systems through simplified models.

Our work leads to the following key conclusions.

- Joint occurrence of precipitation and dissolution is tightly coupled with the existence of preferential flow pathways. Conductivity increase due to the dissolution reaction along such paths leads to enhance particle migration along these. The dominance of preexisting preferential flow regions on the (reactive) transport pattern across the field is therefore further reenforced and self-sustained across time. At the same time, diffusion promotes displacement of particles, leading to precipitation (and hence a progressive reduction over time of local conductivities) at locations in the proximity of these.

- Reactive processes yield an increase over time of the range of conductivity values across the domain, eventually leading to a widening of the support of solute waiting times and conductivity distributions. The clear separation between regions where dissolution or precipitation takes place is reflected in sample distributions of conductivity which tend to become visibly left skewed with time, a feature which is associated with precipitation taking 
place in low conductivity cells located in the proximity of existing preferential flow pathways.

- Solute mass weighted conductivity and velocity distributions are at the basis of our characterization of the parameters of a TPL model and enable us to capture effective (upscaled) non-Fickian transport behaviors. With the progress of precipitation/dissolution reactions, transport shifts towards an increasingly acute non-Fickian effective behavior (see Figure 2 and Table 1). The latter is then seen as a direct outcome of the documented feedbacks between transport and reactions taking place in heterogeneous porous media. The evolution of transport parameters towards a pronounced non-Fickian behavior is associated with only minor changes of the mean and variance of log-conductivity values. This result is consistent with the conceptual picture that the tails of flux and hydraulic conductivity distributions carry critical information to characterize transport while displaying only a minor effect on low order statistics associated with these quantities. Our results suggest that this feature must be acknowledged to properly characterize transport in the presence of precipitation/dissolution.

- We observe the emergence of an asymptotic regime in highly heterogeneous systems, where the (averaged) solute velocity attains a constant value even in the presence of reaction. This suggests the occurrence of an equilibrium state between reactive processes and transport under the flow conditions analyzed. This regime is attained because the effects of locally occurring precipitation and dissolution balance each other at the overall scale of the system, so that the ensuing (ensemble-averaged) solute velocity remains unaffected. The time required to attain such an asymptotic state increases with decreasing initial heterogeneity of the conductivity field, thus suggesting that pre-asymptotic behaviors may be more relevant in initially homogeneous systems.

Table 1. Values of mean and variance of unweighted and weighted log-conductivity distributions and estimated parameters of the effected TPL model obtained through calibration of (6) against the computed distributions of particle waiting times in the domain cells. Results are listed for the three values of the initial log-conductivity variance $\left(\sigma_{0}^{2}\right)$ and are obtained from non-reactive transport simulations performed across conductivity fields resulting from reactive transport simulations at selected times.

\begin{tabular}{|c|c|c|c|c|c|c|c|c|c|}
\hline & \multicolumn{3}{|c|}{$\sigma_{0}^{2}=1$} & \multicolumn{3}{c|}{$\sigma_{0}^{2}=3$} & \multicolumn{3}{c|}{$\sigma_{0}^{2}=5$} \\
\hline$t[\mathrm{~min}]$ & 0 & 10 & 20 & 0 & 10 & 20 & 0 & 10 & 20 \\
\hline $\bar{y}$ & 0.15 & 0.16 & 0.17 & 0.45 & 0.46 & 0.46 & 0.66 & 0.67 & 0.69 \\
\hline$\overline{n y}$ & 0.32 & 0.33 & 0.35 & 0.83 & 0.84 & 0.86 & 1.2 & 1.19 & 1.21 \\
\hline$\sigma^{2}\left(y_{i j}\right)$ & 0.97 & 1.2 & 1.37 & 2.8 & 3.1 & 3.26 & 4.6 & 4.96 & 5.11 \\
\hline$\sigma^{2}\left(n y_{i j}\right)$ & 0.99 & 1.21 & 1.41 & 2.8 & 3.07 & 3.21 & 4.6 & 4.85 & 5 \\
\hline$\beta$ & 2 & 1.4 & 1.05 & 1.7 & 1.2 & 0.95 & 1.4 & 0.8 & 0.6 \\
\hline$t_{1}$ & 0.1 & 0.09 & 0.06 & 0.1 & 0.07 & 0.03 & 0.08 & 0.03 & 0.01 \\
\hline $\log _{10}\left(t_{2}\right)$ & 1 & 2.5 & 2.8 & 1.5 & 2.2 & 2.9 & 1.6 & 2.5 & 3 \\
\hline
\end{tabular}


https://doi.org/10.5194/hess-2021-238

Hydrology and

Preprint. Discussion started: 17 May 2021

(c) Author(s) 2021. CC BY 4.0 License.
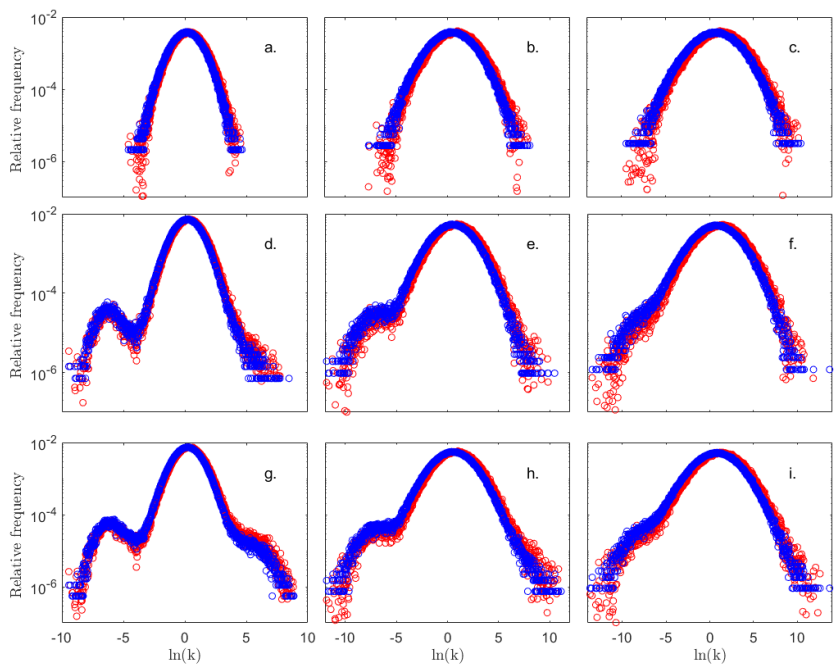

Figure 1. Relative frequency distributions $f\left(y_{i j}\right)$ (blue circles) and $f\left(n y_{i j}\right)$ (red circles) for a tracer test performed on the conductivty field prior to reaction and those associated with reactive simulation times of 10 and 20 minutes. Results correspond to $\sigma_{0}^{2}=1,3,5$ (left, middle and right columns, respectively) and to $t=(a-c) 0,(d-f) 10$, and $(g-i) 20$ min. Mean and variance of these distributions are listed in Table 1.
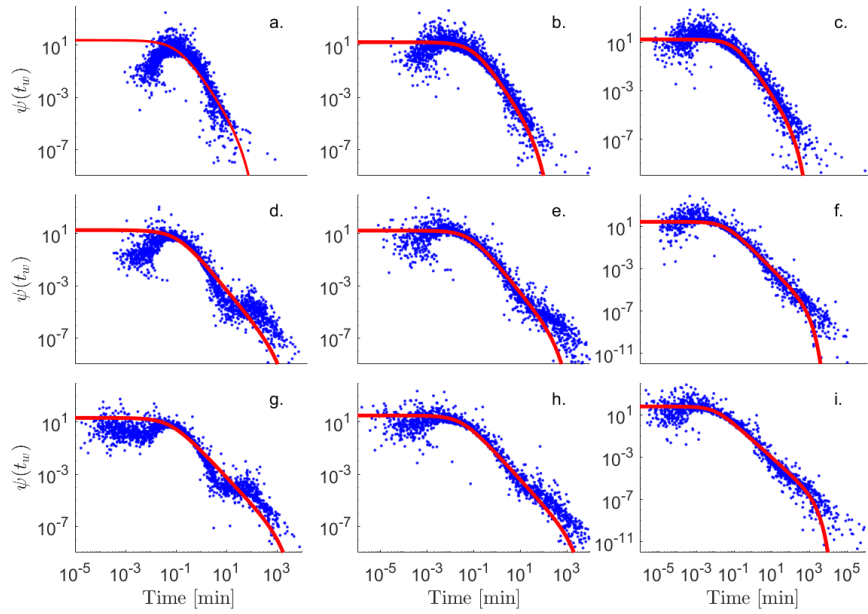

Figure 2. Sample and modeled probability density function $\psi\left(t_{w}\right)$ of particle waiting times for a tracer test performed on the conductivty field prior to reaction those associated with reactive simulation times of 10 and 20 minutes. Results correspond to $\sigma_{0}^{2}=$ 1, 3, 5 (left, middle and right columns, respectively) and $t=(a-c) 0,(d-f) 10 \mathrm{~min}$, and $(\mathrm{g}-\mathrm{i}) 20 \mathrm{~min}$. Values of TPL parameters estimated by calibrating model (6) on the sample distributions are listed in Table 1. 
https://doi.org/10.5194/hess-2021-238

Hydrology and

Preprint. Discussion started: 17 May 2021

(c) Author(s) 2021. CC BY 4.0 License.

430
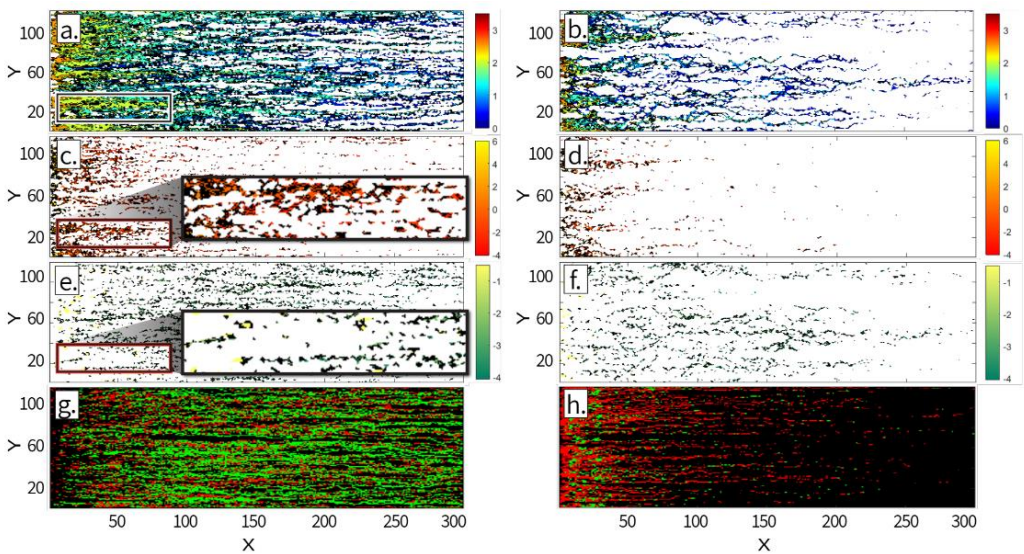

Figure 3. Heat map representing $(a, b) \log _{10}\left(n_{\text {Rij }}\right)$, i.e., the number of $h$ particles visiting each cell for $\sigma_{0}^{2}=1$ and 5 , respectively, and $(c-f)$ relative change in hydraulic conductivity at time $t=20$ min with respect to the initially generated values for $\sigma_{0}^{2}=1(c$ and e) and $\sigma_{0}^{2}=5$ ( $d$ andf). Panels $c$ and d display positive changes in conductivity with respect to the initial field, while panels e and $f$ display negative changes in conductivity, both positive and negative changes being represented in log-scale. Results correspond to a selected realizaton of the the log-conductivity fields. The highlighted box illustrates the separation between regions where

precipitation or dissolution take place. Panels $g$ and $h$ display cells associated with a net increase (green) and decrease (red) of conductivity for cells outside ( $g$ ) or within the $P F(h)$, for $\sigma_{0}^{2}=1$.
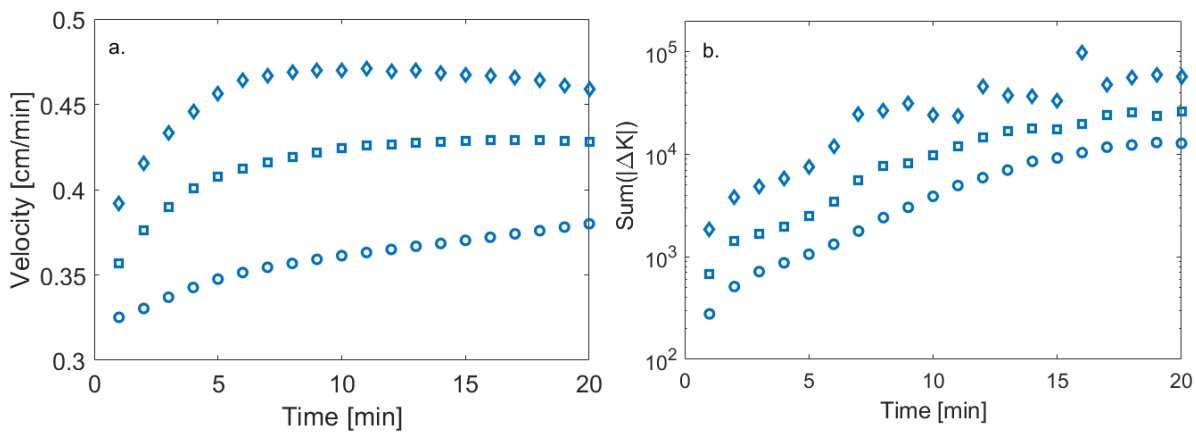

Figure 4. Temporal evolution of (a) the weighted mean velocity $\overline{n_{R} v}$ (open symbols) and (b) the sum of all conductivity changes over 1 minut. Results correspond to $\sigma_{0}^{2}=1$ (circles), 3 (squares), and 5 (diamond). Please, check all symbols along the axes are defined exactly with the same terminology in the text, ok? 
Ababou, R., McLaughlin, D., Gelhar, LynnW., \& Tompson, AndrewF. B. (1989). Numerical simulation of three-dimensional saturated flow in randomly heterogeneous porous media. Transport in Porous Media, 4(6). https://doi.org/10.1007/BF00223627 Atchley, A. L., Navarre-Sitchler, A. K., \& Maxwell, R. M. (2014). The effects of physical and geochemical heterogeneities on hydro-geochemical transport and effective reaction rates. Journal of Contaminant Hydrology, 165, 53-64. https://doi.org/10.1016/j.jconhyd.2014.07.008 geological formations as a continuous time random walk. Reviews of Geophysics, 44(2), RG2003. https://doi.org/10.1029/2005RG000178

Berkowitz, B., Dror, I., Hansen, S. K., \& Scher, H. (2016). Measurements and models of reactive transport in geological media: REACTIVE TRANSPORT MEASURES AND MODELS. Reviews of Geophysics, 54(4), 930-986. https://doi.org/10.1002/2016RG000524

Cabeza, Y., Hidalgo, J. J., \& Carrera, J. (2020). Competition Is the Underlying Mechanism Controlling Viscous Fingering and Wormhole Growth. Geophysical Research Letters, 47(3). https://doi.org/10.1029/2019GL084795

462 Ceriotti, G., Guadagnini, L., Porta, G., \& Guadagnini, A. (2018). Local and Global Sensitivity 463 Analysis of Cr (VI) Geogenic Leakage Under Uncertain Environmental Conditions. Water 464 Resources Research, 54(8), 5785-5802. https://doi.org/10.1029/2018WR022857 Model of Arsenic Mobility in the Shallow Alluvial Aquifers Near Venice (Italy) Elucidated Through Machine Learning and Geochemical Modeling. Water Resources Research, 56(9). https://doi.org/10.1029/2019WR026234

469 Dentz, M., Gouze, P., \& Carrera, J. (2011). Effective non-local reaction kinetics for transport in 470 physically and chemically heterogeneous media. Journal of Contaminant Hydrology, 120-121, 471 222-236. https://doi.org/10.1016/j.jconhyd.2010.06.002

472 Edery, Y. (2020). The topological origin of anomalous transport: Persistence of $\{\backslash$ beta $\}$ in the face 473 of varying correlation length. ArXiv:1906.07061 [Physics]. Retrieved from 474 http://arxiv.org/abs/1906.07061

475 Edery, Y., Scher, H., \& Berkowitz, B. (2011). Dissolution and precipitation dynamics during dedolomitization: REACTION DYNAMICS DURING DEDOLOMITIZATION. Water Resources Research, 47(8). https://doi.org/10.1029/2011WR010551

Edery, Y., Guadagnini, A., Scher, H., \& Berkowitz, B. (2014). Origins of anomalous transport in heterogeneous media: Structural and dynamic controls. Water Resources Research, 50(2), 14901505. https://doi.org/10.1002/2013WR015111

Edery, Y., Porta, G. M., Guadagnini, A., Scher, H., \& Berkowitz, B. (2016). Characterization of Bimolecular Reactive Transport in Heterogeneous Porous Media. Transport in Porous Media, 115(2), 291-310. https://doi.org/10.1007/s11242-016-0684-0

Edery, Y., Geiger, S., \& Berkowitz, B. (2016). Structural controls on anomalous transport in fractured porous rock: ANOMALOUS TRANSPORT IN FRACTURED POROUS ROCK. Water Resources Research, 52(7), 5634-5643. https://doi.org/10.1002/2016WR018942

Erol, S., Fowler, S. J., Harcouët-Menou, V., \& Laenen, B. (2017). An Analytical Model of Porosity-Permeability for Porous and Fractured Media. Transport in Porous Media, 120(2), 327358. https://doi.org/10.1007/s11242-017-0923-z

Garing, C., Gouze, P., Kassab, M., Riva, M., \& Guadagnini, A. (2015). Anti-correlated PorosityPermeability Changes During the Dissolution of Carbonate Rocks: Experimental Evidences and Modeling. Transport in Porous Media, 107(2), 595-621. https://doi.org/10.1007/s11242-015-0456- 
Golfier, F., Zarcone, C., Bazin, B., Lenormand, R., Lasseux, D., \& Quintard, M. (2002). On the ability of a Darcy-scale model to capture wormhole formation during the dissolution of a porous medium. Journal of Fluid Mechanics, 457, 213-254. https://doi.org/10.1017/S0022112002007735 Gómez-Hernández, J. J., \& Journel, A. G. (1993). Joint Sequential Simulation of MultiGaussian Fields. In A. Soares (Ed.), Geostatistics Tróia '92 (Vol. 5, pp. 85-94). Dordrecht: Springer Netherlands. https://doi.org/10.1007/978-94-011-1739-5_8 Krauskopf, K. B., \& Bird, D. K. (1967). Introduction to geochemistry (Internat.3Rev.ed). Le Borgne, T., Dentz, M., \& Carrera, J. (2008). Lagrangian Statistical Model for Transport in Highly Heterogeneous Velocity Fields. Physical Review Letters, 101(9), 090601. https://doi.org/10.1103/PhysRevLett.101.090601

Liu, P., Yao, J., Couples, G. D., Ma, J., \& Iliev, O. (2017). 3-D Modelling and Experimental Comparison of Reactive Flow in Carbonates under Radial Flow Conditions. Scientific Reports, 7(1), 17711. https://doi.org/10.1038/s41598-017-18095-2

Menke, H. P., Reynolds, C. A., Andrew, M. G., Pereira Nunes, J. P., Bijeljic, B., \& Blunt, M. J. (2018). 4D multi-scale imaging of reactive flow in carbonates: Assessing the impact of heterogeneity on dissolution regimes using streamlines at multiple length scales. Chemical Geology, 481, 27-37. https://doi.org/10.1016/j.chemgeo.2018.01.016

Morse, J. W., \& Mackenzie, F. T. (1993). Geochemical constraints on CaCO3 transport in subsurface sedimentary environments. Chemical Geology, 105(1-3), 181-196. https://doi.org/10.1016/0009-2541(93)90125-3

Muljadi, B. P., Bijeljic, B., Blunt, M. J., Colbourne, A., Sederman, A. J., Mantle, M. D., \& Gladden, L. F. (2018). Modelling and upscaling of transport in carbonates during dissolution: Validation and calibration with NMR experiments. Journal of Contaminant Hydrology, 212, 85-95. https://doi.org/10.1016/j.jconhyd.2017.08.008

Noiriel, C., \& Daval, D. (2017). Pore-Scale Geochemical Reactivity Associated with CO 2 Storage: New Frontiers at the Fluid-Solid Interface. Accounts of Chemical Research, 50(4), 759-768. https://doi.org/10.1021/acs.accounts.7b00019

Rege, S. D., \& Fogler, H. S. (1989). Competition among flow, dissolution, and precipitation in porous media. AIChE Journal, 35(7), 1177-1185. https://doi.org/10.1002/aic.690350713

Riva, M., Guadagnini, A., Neuman, S. P., Janetti, E. B., \& Malama, B. (2009). Inverse analysis of stochastic moment equations for transient flow in randomly heterogeneous media. Advances in Water Resources, 32(10), 1495-1507. https://doi.org/10.1016/j.advwatres.2009.07.003

Saripalli, K. P., Meyer, P. D., Bacon, D. H., \& Freedman, V. L. (2001). Changes in Hydrologic Properties of Aquifer Media Due to Chemical Reactions: A Review. Critical Reviews in Environmental Science and Technology, 31(4), 311-349. https://doi.org/10.1080/20016491089244 Singurindy, O., \& Berkowitz, B. (2004). Dedolomitization and flow in fractures. Geophysical Research Letters, 31(24), L24501. https://doi.org/10.1029/2004GL021594 